\title{
An Unexpected Use of the Shiny Package for $\mathbf{R}$
}

\section{Fu-Sheng Chou, MD, PhD}

\begin{abstract}
History
The R statistical language has been around since 1995. Ross Ihaka and Robert Gentleman first developed it in 1991 at the University of Auckland in New Zealand. The project became publicized in 1993. R is open-source software under the GNU General Public License. This is probably why academicians and statisticians widely use $\mathrm{R}$ for data mining, analysis, and research communication. The powerful engine for graphics rendering in $\mathrm{R}$ distinguishes itself from other commonly used software packages such as Microsoft@ Excel in data plotting and graphic annotation. The most recent stable update at the time of this article is version 4.0.3 and is available for download from multiple Comprehensive R Archive Network (CRAN) mirror sites. (1)
\end{abstract}

RStudio ${ }^{\circ}$ was founded by J. J. Allaire in 2009 , with a mission to create free and open-source software for data science, scientific research, and technical communication. As of 2019, RStudio $®$ has become a Public Benefit Corporation. RStudio ${ }^{\circledR}$ produced its first integrative development environment (IDE) for $R$ in 2011. The open-source edition of the RStudio IDE is licensed under AGPL v3 and is freely available to anyone. The most recent stable release of RStudio IDE at the time of this article is version 1.3. (2)

\section{"The open-source edition of the RStudio IDE is licensed under AGPL v3 and is freely available to anyone. The most recent stable release of RStudio IDE at the time of this article is version 1.3. (2)"}

\section{R packages}

There are now over 16,000 packages for $R$ that are freely available for download to facilitate specific areas of workflow, analysis, statistical modeling, plotting, and result distribution.(3) In addition to developing IDE, RStudio ${ }^{\circledR}$ is also heavily involved in R package development to improve user experience. One area of their focus is research communication. RStudio $₫$ has created three packages in this regard, namely, Shiny, rmarkdown, and flexdashboard, all with a unifying mission to facilitate information distribution and communication.(4) Shiny and rmarkdown are two critical packages developed by RStudio ${ }^{\circ}$ that were used to develop the new Neonatology Today website (or you can call it a web application, or a webApp). Shiny lets developers build webApps that are intrinsically interactive using R syntax.(5) These interactive Shiny webApps allow researchers to communicate the full spectrum of their results with the audience based on the audience's input. On the other hand, rmarkdown allows the audience to generate final reports in a variety of formats (PDF, HTML, Word file, etc.).(6)

\section{Structure of the Neonatology Today webApp}

The new Neonatology Today webApp is built natively in $\mathrm{R}$ and Rstudio ${ }^{\circledR}$ IDE using the Shiny package and others. Figure 1 outlines the structure of the Neonatology Today webApp. The webApp consists of two parts: 1) content presentation and 2) user data en- try, including the Online Manuscript Submission System and the Online Manuscript Review System. Notably, additional webApps were developed, allowing the management team to update the content and control the flow of the submitted manuscripts.

\section{"Notably, additional webApps were developed, allowing the management team to update the content and control the flow of the submitted manuscripts."}

\section{Content presentation}

The webApp allows the readers to access the journal content in both PDF or HTML formats. The PDFs are available for articles from January 2019 to current, and HTML formats are available for selected articles from July 2020 . The webApp also contains a search function for articles in the HTML format. The search function provides a great example of the reactivity of a Shiny webApp.

Besides journal articles, additional features were created to enrich reader experience, including a running Announcement carousel, an Events box, and a New \& Views box accessible from the main page. The well-appreciated author artwork in the original PDF format is also available to the readers in a carousel, alongside the editors' information.

The front page is designed with mobile device access in mind. The content display should be equally visually satisfying when viewed using major mobile browsers on mobile devices.

\section{Online Manuscript Submission System}

The Online Manuscript Submission System was developed with a goal in mind to allow authors to create, save, and submit manuscripts directly within the webApp. The interactive nature of webApps developed using the Shiny package made it an ideal tool for such tasks. With the combined use of the rmarkdown package, the authors can fully concentrate on their manuscripts' content without the need to spend time typesetting their manuscripts before submission.

Figures and tables to be uploaded to the Online Manuscript Submission System are preferably prepared in PDF format, a popular format built into modern operating systems (the Print as PDF function), making the conversion effortless. The PDFs store information such as text, font type, vectorized graphs, and pixelated images in one file. This is different from other manuscript submission systems, where different file formats are requested for different figure types, and image resolution was always a concern after image file type conversion.

Before submission, the compiled manuscript and cover letter files are downloaded as PDFs thanks to the rmarkdown package for review. Once approved by the authors, the manuscript and the cover letter may be ready for submission.

The Online Manuscript Submission System is available for public testing. Please note that we have discovered that it 
does not function properly in the Firefox browser.

"A more sophisticated webApp can be created to guide the clinical decision, provided the background statistical model has a good predictive value. Shiny webApp developers may be concerned about HIPAA compliance, but there are ways to avoid violating the regulations. "
Online Manuscript Review System

Like the Online Manuscript Submission System, the Online Manuscript Review System allows the reviewers to interact with the webApp to enter their comments and feedback. The System will record the comments. Once the comments are submitted, the System will generate a certificate for the reviewer, thanks to the rmarkdown package.

With the abundant amount of available packages for developers to use "behind the scene," the potential of a Shiny webApp is enormous. Imagine having a Shiny webApp with a user interface for parameter entry and a machine learning-based model on the server-side to calculate clinical outcome prediction. The machine learning-based model hiding in the background may have been developed natively in $\mathrm{R}$ using the famous ranger random forests

\section{Neonatology Today webApp}

\section{Content Access}

- PDF for the whole issue

- PDF for each article

- HTML for each article

Search function - Keyword, Title, Abstract, Full text, Authors

- News \& Views

Highlights of interesting papers from peer journals in

Neonatal-Perinatal Medicine

- Announcement \& Events

\section{Manuscript Process}

- Submission

- Reviewers' feedback

\section{Ancillary Functions}

- Subscription

- Artwork display

- Leaderboard Ad management 
modeling package. The newly entered parameter data is stored in a SQL database using the odbc package and is called periodically for use to re-train the model. The $\mathrm{R}$ statistical language does it all, seamlessly, using the same syntax. Most importantly, the syntax is intuitive to people like me who have no computer language experience and do not know how computers work inside metal cases.

In my opinion, simple Shiny webApps can be created for routine clinical data collection and reporting, standardized scoring system development, clinical protocol reminders, real-time quality improvement project reporting. A more sophisticated webApp can be created to guide the clinical decision, provided the background statistical model has a good predictive value. Shiny webApp de-

\section{Neonatology Today's Digital Presence}

Neonatology Today's now has a digital presence. Although officially still in beta, the site is operational now and defines the future look of our digital web presence. By clicking on this https://www. neonatologytoday.org/web/., researchers can download individual manuscripts both in digital format and as part of the orginal PDF (print journal). While the PDF version of Neonatology Today will continue in its present form, we envision that the entire website will be migrated to this format in the next several months. We encourage you to take a look, "kick the wheels," and let us know where we still need to improve.. We are working towards making the website more functional for subscribers, reviewers, authors and anyone else. Although we have not yet applied for inclusion in the National Library of Medicine Database (Pub-Med), this new format meets several of the important metrics for this ultimate goal.

As we indicated last month, we look forward to a number of new features as well.

1. An online submission portal: Submitting a manuscript online will be easier than before. Rather than submitting by email, we will have a devoted online submission portal that will have the ability to handle any size manuscript and any number of graphics and other support files. We will have an online tracking system that will make it easier to track manuscripts in terms of where they are in the review process.

2. Reviewers will be able to review the manuscript online. This portal will shorten the time from receipt of review to getting feedback to the submitting authors.

3. An archive search will be available for journals older than 2012.

4. A new section called news and views will enable the submission of commentary on publications from other journals or news sources. We anticipate that this will be available as soon as the site completes the beta phase

5. Sponsors will be able to sign up directly on the website and submit content for both the digital and PDF issues of Neonatology Today.

Neonatology Today will continue to promote our Academic True Open Model (ATOM), never a charge to publish and never a charge to subscribe.Please see the next page for details

Please find a preview of our new interface just to the left of this column.

If there are any questions about the new website, please email Dr. Chou directly at:

fu-sheng.chou@neonatologytoday.net velopers may be concerned about HIPAA compliance, but there are ways to avoid violating the regulations. Data used for analysis in $\mathrm{R}$ are stored in the random-access memory (RAM), which means that, unless you ask $R$ to write the data object into a file on a disk or a flash drive, the data will not be stored permanently. In other words, as long as the private health information is not directly written into a file, there should be minimal concerns for HIPAA violation. A local institutional review board should always be consulted to determine compliance at the institute level.

I would like to thank Dr. Goldstein again for creating this Data Science column for me to share my stories about learning coding in $\mathrm{R}$ and developing shiny webApps. Starting next month, I would like to introduce you to some simple $R$ syntax and show you how to develop simple webApps that may assist you with some of your routine workflows in the NICU. I plan to show you how to create a modified Finnegan scoring webApp to assess the severity of neonatal abstinence syndrome and opioid withdrawal.

More to come. Thanks for reading this post!

\section{References:}

1. Hornik K. The Comprehensive R Archive Network. WIREs Comp Stat. 2012;4(4):394-398. doi:10.1002/wics.1212

2. RStudio. Accessed November 16, 2020. https://rstudio. com/products/rstudio/

3. Contributed Packages. Accessed November 16, 2020 https://cran.r-project.org/web/packages/

4. R Packages. Accessed November 16, 2020. https://rstudio. com/products/rpackages/

5. Web Application Framework for $R[R$ package shiny version 1.5.0]. Published online June 23, 2020. Accessed November 16, 2020. https://cran.r-project.org/web/packages/shiny/ index.html

6. Dynamic Documents for $R[R$ package rmarkdown version 2.5]. Published online October 21, 2020. Accessed November 16, 2020. https://cran.r-project.org/web/packages/ rmarkdown/index.html

Disclosure: The author identifies no conflict of interest

NT

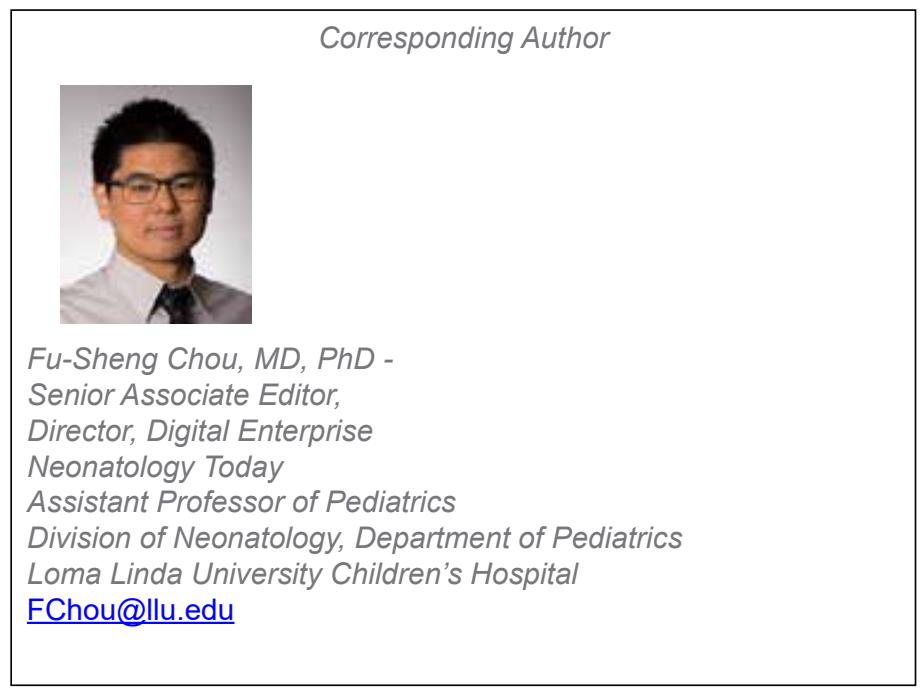

